Dome C Astronomy and Astrophysics Meeting

M. Giard, F. Casoli and F. Paletou (eds)

EAS Publications Series, 14 (2005) 245-250

\title{
CLOVER EXPERIMENT: THE RECEIVER BLOCK
}

G. Pisano ${ }^{1}$, P.A.R. Ade $^{1}$, C. Calderon ${ }^{1}$, A.D. Challinor ${ }^{2}$, P. De Bernardis ${ }^{3}$, L. Dunlop ${ }^{2}$, W.K. Gear ${ }^{1}$, Y. Giraud-Héraud ${ }^{4}$, D.J. Goldie ${ }^{2}$, K.J.B. Grainge ${ }^{2}$, K.G. Isaak ${ }^{1}$, B. Johnson ${ }^{1}$, M.E. Jones ${ }^{2}$, A.N. Lasenby ${ }^{2}$, B. Maffei ${ }^{1}$, P.D. Mauskopf ${ }^{1}$, S.J. Melhuish ${ }^{1}$, A. Orlando ${ }^{1}$, L. Piccirillo ${ }^{1}$, A.C. Taylor ${ }^{2}$, S. Withington ${ }^{2}$ and G. Yassin ${ }^{2}$

\begin{abstract}
The $\mathrm{C}_{l}$ OVER instrument (described elsewhere in this volume) is being built to measure the $B$-mode polarisation of the Cosmic Microwave Background. Each of the 256 pixels is made up a pseudocorrelation receiver that can be realised using either waveguide or microstrip technology. In this work we present a design study for a possible waveguide-based solution. Each of the individual components has been optimised using electromagnetic finite-element modelling software (HFSS).
\end{abstract}

\section{Introduction}

The primary goal of the $\mathrm{C}_{l}$ OVER experiment is to measure the polarisation of the Cosmic Microwave Background. To achieve this goal, it is necessary to understand in detail the instrumental systematics and to minimise them. $\mathrm{C}_{l}$ OVER comprises three, independent telescopes, operating at 90, 150 and $220 \mathrm{GHz}$ and will be located at DOME-C in Antarctica. Each telescope is broken down into four, separate $8 \times 8$ arrays of feed horns, each of which is pointed at the same region of the sky. The signal incident on each horn is processed in a pseudo-correlation receiver. The outputs from the corresponding pixel in the four arrays are summed, incoherently, before being detected by a TES bolometer. There are therefore a total of 256 horns per telescope, yet a total of only 64 pointings on the sky. The sensitivity, however, is equivalent to 256 distinct pixels (Taylor et al. 2004; Yassin et al. 2004; Maffei et al. 2004). In this paper we present a brief description

\footnotetext{
${ }^{1}$ Department of Physics and Astronomy University of Cardiff, UK

2 Cavendish Astrophysics, University of Cambridge, UK

${ }^{3}$ Università La Sapienza, Roma, Italy

${ }^{4}$ Collège de France, Paris, France
} 
of the conceptual design of the pseudo-correlation receiver which is at the core of ClOVER, and describe a wave-guide-based implementation.

\section{The Pseudo-Correlation Receiver}

Shown in Figure 1 is a schematic of the pseudo-correlation receiver. The signal incident on each horn is split into its two, independent, linear polarisation states by an Ortho-Mode Transducer (OMT). A 90-degrees hybrid is used to convert the polarisation state of the signals from linear to circular. Phase shifters are then used to introduce a differential phase shift between the two arms of the receiver, $\varphi=\varphi_{1}-\varphi_{2}$. These signals are then recombined coherently using a second 90-degrees hybrid. The Stokes parameters, $I, U$ and $Q$ are encoded in the two correlated outputs in the following way:

$$
\begin{aligned}
& D_{1}=\frac{1}{2}(I-Q \cdot \cos \Phi-U \cdot \sin \Phi) . \\
& D_{2}=\frac{1}{2}(I+Q \cdot \cos \Phi+U \cdot \sin \Phi) .
\end{aligned}
$$

Each pixel is therefore sensitive to $I, U$ and $Q: Q$ and $U$ can be recovered by modulating the differential phase in the pseudo-correlation receiver, while $I$ is recovered by scanning the telescope. Explicitly, by switching the phase, $\Phi$, between -90 and +90 degrees the difference in the signals in the two arms, $\left(D_{2}-D_{1}\right)$, will measure $U$, as under these circumstances the net contribution from $Q$ will be zero. Conversely, by switching the phase between 0 and 180 degrees, the net contribution from $U$ is zero, and $Q$ can be measured. Note that the parameter $I$, which will be sensitive to instrumental drifts, is not modulated by the phase switching.

\section{A Pseudo-Correlation Receiver Design Using Waveguide}

Shown in the right hand side of Figure 1 is a design for the pseudo-correlation receiver based on waveguide technology. The symmetry of all components allows the waveguide to be split into two halves. This simplifies the machining without overly affecting performance and means that CNC machining techniques as well as spark-erosion and laser-technology can be used to realise the fine detail required in several of the components.

\subsection{The Finline Ortho-Mode Transducer}

An Ortho-Mode Transducer (OMT) is designed to separate two orthogonal linearly polarised signals travelling in a waveguide. The requirements for the $\mathrm{C}_{l} \mathrm{OVER}$ OMTs (return loss: $\sim-20 \mathrm{~dB}$; cross-polarisation: $\sim-30 \mathrm{~dB}$ and band-width: $\sim 25 \%$ can be met using OMTs based on finline technology (Chattopapdhyay et al. 1999), (Robertson 1956), (Groppi et al. 2004). In a finline OMT realisation, two diametrically opposing thin, tapered, metallic fins are placed into a square cross-section 


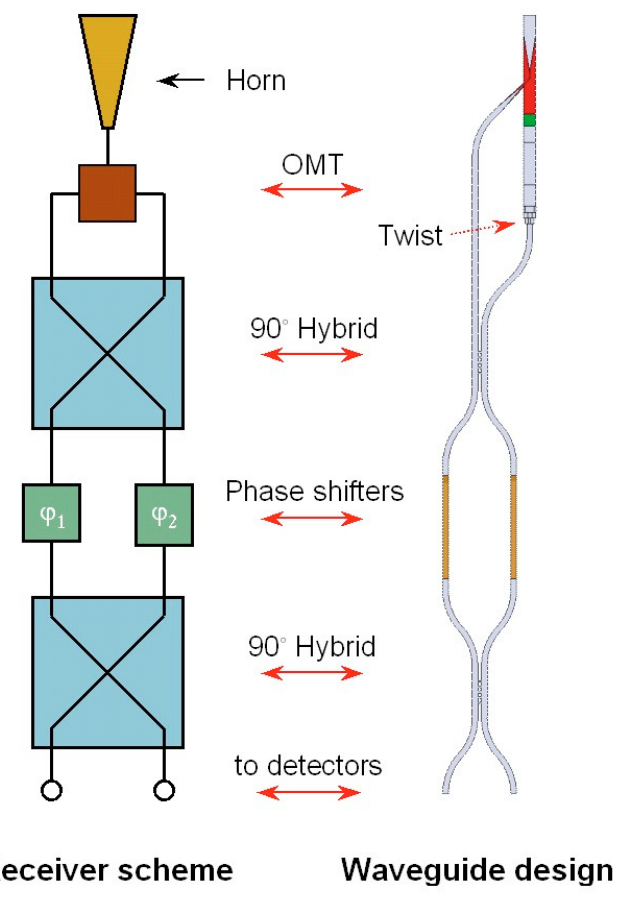

Fig. 1. The pseudo-correlation receiver scheme: schematic and waveguide design.

waveguide (Fig. 2) The mode with the electric field parallel to the fins is gradually transformed to a finline mode which is confined between the fins in the central region of the waveguide. This mode is subsequentely extracted from the waveguide by curving the finline and bringing it out through the side wall. The mode is subsequently re-launched in a waveguide with rectangular cross-section. The mode with the orthogonal polarisation passes along the guide largely unperturbed: the cross-section of the waveguide is tapered down to a horizontal, rectangular waveguide (Fig. 2). A broadband 90-degrees waveguide twist (Rosenberg et al. 2001) is required to align the two waveguide outputs in order that both polarisation modes travel in parallel rectangular waveguides. The finline taper has been designed using mode-matching techniques, while the optimisation of the whole OMT has been achieved using finite element analysis (HFSS). The finline will be fabricated using photolithographic techniques.

\subsection{A Branch-Line 90-Degrees Hybrid}

A 90 degrees hybrid is a four-port directional coupler in which the power incident on any one port is divided equally between two other ports $(-3 \mathrm{~dB})$ with a 90-degrees phase difference between them; the fourth port is isolated. The coupler 


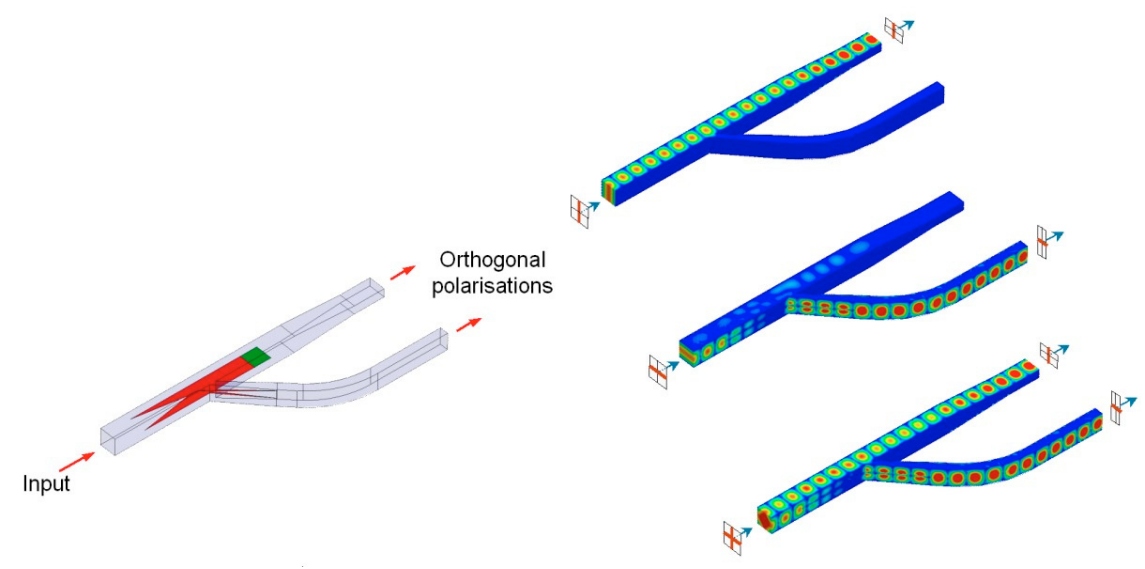

Fig. 2. The fin-line orthomode transducer: schematic of the physical design and simulations of the response (HFSS).

to be used here is of a branch-line design, in which two waveguides are interconnected by multiple branch waveguides between the walls. E-plane branchline couplers have been shown (Andohet al. 2003) to have excellent performance: $(S 12, S 13)=3.0 \pm .3 \mathrm{~dB},(S 11, S 14)<-25 \mathrm{~dB}$ across a $25 \%$ bandwidth. Shown in Figure 3 are the results of HFSS simulations of the hybrid, which illustrate the field distribution in the branchline region (top panel) of the coupler, and in the waveguides (lower panel).

\subsection{Mechanical Phase Shifter}

A phase-shifter is used to add a specific phase delay to a signal. Our design is based on a double dielectric-slab-filled waveguide (Arndt et al. 1985). Two dielectric slabs with linear tapered sections are positioned close to the sidewalls of the waveguide (Fig. 4, upper panel). A phase shift in the range of 0-360 degrees relative to that of an empty waveguide can be achieved by changing the slab displacement relative to the waveguide walls. Near-constant differential phase-shifts can be achieved over a wide range of frequencies using this design. The phase can be modulated, as required for the correlation receiver design, by moving periodically the dielectric slabs using cryogenic piezo-electric actuators. The present model allows phase-shifts in the range of $0 \mathrm{deg} \leq \Delta \Phi \leq 180 \mathrm{deg}$, with a return loss of better than $30 \mathrm{~dB}$ with a maximum phase error of $\Delta \Phi= \pm 3.8$ degrees over a $20 \%$ bandwidth. The simulations shown in Figure 4 (lower panel) illustrate how the waveguide wavelength, and so phase, changes as a function of slab displacement. 

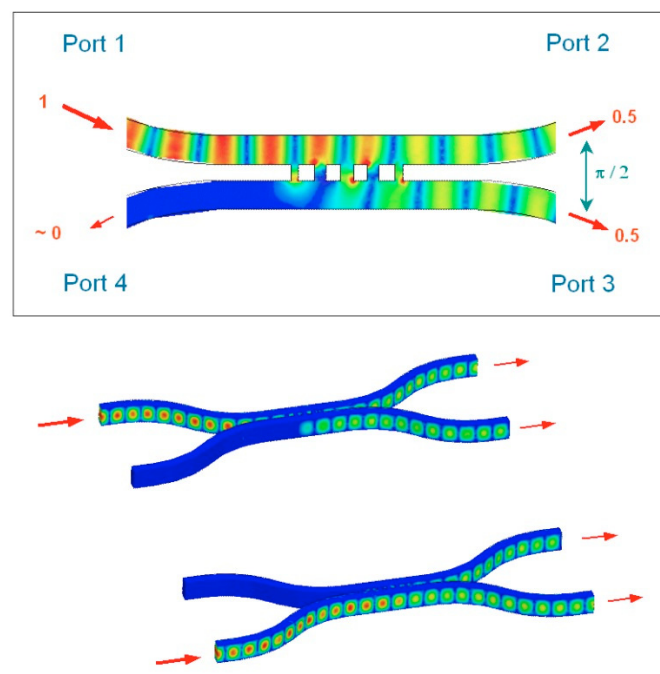

Fig. 3. HFSS simulations of the response of the branch-line 90-degrees hybrid design.

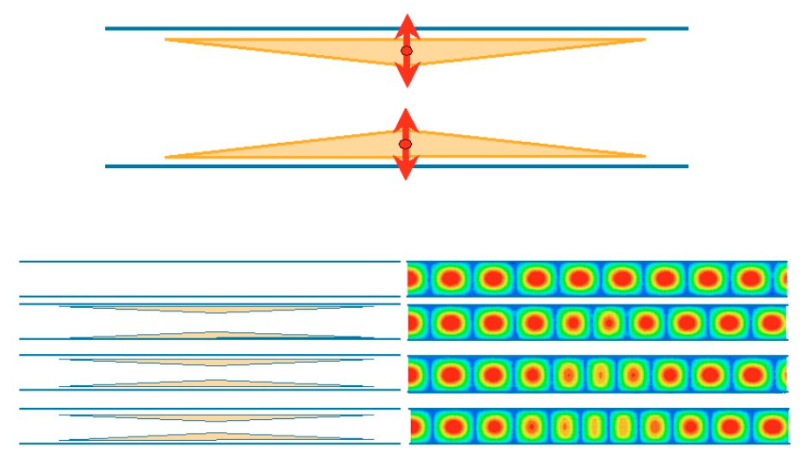

Fig. 4. The double dielectric slab phase shifter: schematic (top panel) and simulated response (lower panel).

\section{Conclusions}

We have presented a solution for the $\mathrm{C}_{l}$ OVER pseudo-correlation receiver. Each of the components in the receiver is based around a design that has been shown to work at other frequencies. We have scaled, adapted and individually optimised the parts to realise receiver chains that will operate up to $220 \mathrm{GHz}$. A simulation of the 


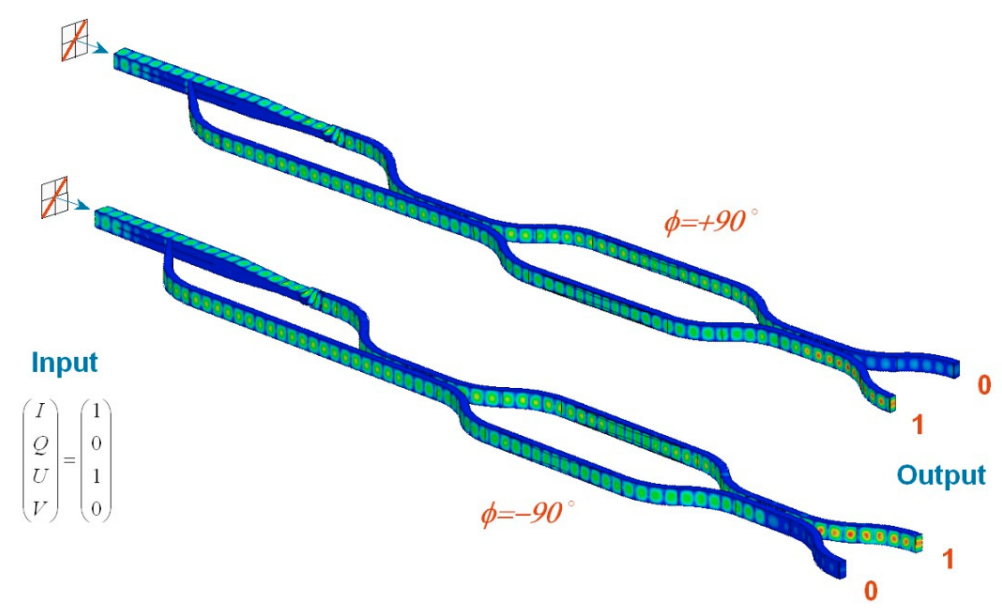

Fig. 5. A simulation of the overall response of the pseudo-correlation receiver using HFSS.

complete receiver is shown in Figure 5, where the $U$ Stokes parameter is modulated between two detectors by means of a phase modulation $\Phi= \pm 90$ degrees.

We acknowledge the support of the Particle Physics and Astronomy Research Council of the UK in this work.

\section{References}

High Frequency Structure Simulator (HFSS) web site: www.ansoft.com

Taylor, A.C., et al. 2004, Proc. XXXIXth Rencontres de Moriond: Exploring the Universe (La Thuile, Italy)

Yassin, G., et al. 2004, Proc. Fifteenth International Symposium on Space $\mathrm{THz}$ Technology, Amherst, MA, USA

Maffei, B., et al. 2004, this volume

Robertson, S.D. 1956, IRE Trans. Micr. Theo. Tech., MTT-4, 263

Chattopapadhyay, G., et al. 1999, IEEE Micr. Guid. Wav. Lett., 9, 339

Groppi, C.E., et al. 2004, Proc. Fifteenth International Symposium on Space $\mathrm{THz}$ Technology, Amherst, MA, USA

Rosenberg, U., et al. 2001, Proc. 31st European Microwave Conf, London UK, 1, 205

Andoh, H., et al. 2003, Int. J. Infr. Mill. Wav., 24(5), 773

Arndt, F., et al. 1985, IEEE Trans. Micr. Theo. Tech., MTT-33, 5, 373 